\title{
ON $n$-DIMENSIONAL LORENTZ MANIFOLDS ADMITTING AN ISOMETRY GROUP OF DIMENSION $n(n-1) / 2+1$
}

\author{
HIROO MATSUDA
}

\begin{abstract}
We classify connected $n$-dimensional Lorentz manifolds admitting an isometry group of dimension $n(n-1) / 2+1$ with compact isotropy subgroup $(n \geq 5)$.
\end{abstract}

1. A connected $n$-dimensional Riemannian manifold admitting a connected closed isometry group of dimension $n(n-1) / 2+1(n \geq 4)$ was completely determined by Yano [8], Ishihara [1], and Obata [5] (cf. Kobayashi [2]). We consider the classification problem of Lorentz manifolds. Each of the following examples is a connected $n$-dimensional Lorentz manifold $M$ admitting a connected isometry group $G$ of dimension $n(n-1) / 2+1$.

EXAMPLE. (i) $M=\mathbf{R} \times N$ with metric $-d t^{2}+d s_{N}^{2}$, and $G=\mathbf{R} \times I^{0}(N)$.

(ii) $M=S^{1} \times N$ with metric $-d \theta^{2}+d s_{N}^{2}$, and $G=S^{1} \times I^{0}(N)$.

(iii) $M=\mathbf{R} \times \mathbf{P}$ with metric $-d t^{2}+d s_{\mathbf{P}}^{2}$, and $G=\mathbf{R} \times I^{0}(\mathbf{P})$.

(iv) $M=S^{1} \times \mathbf{P}$ with metric $-d \theta^{2}+d s_{\mathbf{P}}^{2}$, and $G=S^{1} \times I^{0}(\mathbf{P})$.

(v) $M=U_{n}^{+}=\left\{\left(u_{1}, \ldots, u_{n}\right) ; u_{n}>0\right\}$ with metric

$$
d s_{+}^{2}=\left(d u_{1}^{2}+\cdots+d u_{n-1}^{2}-d u_{n}^{2}\right) /\left(c u_{n}\right)^{2} \quad(c \neq 0),
$$

and $G=I^{0}\left(U_{n}^{+}\right)$(see Nomizu [4]).

(vi) $M=U_{n}^{-}=\left\{\left(u_{1}, \cdots, u_{n}\right) ; u_{n}>0\right\}$ with metric

$$
d s_{-}^{2}=\left(-d u_{1}^{2}+d u_{2}^{2}+\cdots+d u_{n}^{2}\right) /\left(c u_{n}\right)^{2} \quad(c \neq 0),
$$

and $G=I^{0}\left(U_{n}^{-}\right)$(see Matsuda [3]).

Here $N$ is a simply connected $(n-1)$-dimensional Riemannian manifold with metric $d s_{N}^{2}$ of constant curvature and $\mathbf{P}$ is an $(n-1)$-dimensional real projective space with standard metric $d s_{\mathbf{P}}^{2}$. A real line and a circle of a certain radius are denoted by $\mathbf{R}$ and $S^{1}$ respectively. $I^{0}(\cdot)$ denotes the identity component of the full-isometry group of $(\cdot)$.

The purpose of this note is to prove the following theorem.

THEOREM. Let $M$ be a connected $n$-dimensional Lorentz manifold admitting a connected isometry group $G$ of dimension $n(n-1) / 2+1(n \geq 5)$ whose isotropy subgroup at every point is compact. Then $M$ must be one of the spaces (i)-(v).

REMARK 1. The isotropy subgroup of $G$ in the above example is compact except (vi).

REMARK 2. The spaces of (v) and (vi) are not geodesically complete.

Received by the editors May 1, 1985.

1980 Mathematics Subject Classification (1985 Revision). Primary 53C50.

Key words and phrases. Lorentz manifold, isometry group, semi-Riemannian submersion. 
2. Let $(M,\langle\rangle$,$) be a connected n$-dimensional Lorentz manifold with signature $(-,+, \ldots,+)$. Let $G$ be a connected isometry group of $(M,\langle\rangle$,$) and H_{p}$ the isotropy subgroup of $G$ at a point $p \in M$. Then the linear isotropy group $\tilde{H}_{p}=$ $\left\{d h_{p} ; h \in H_{p}\right\}$ acting on $T_{p} M$ is a closed subgroup of $O(1, n-1)$.

LEMMA 1. Ever compact subgroup of $O(1, n-1)$ is conjugate to a subgroup of $O(1) \times O(n-1)$ (cf. Wolf [7]). Especially if $K$ is a compact subgroup of $O(1, n-1)$ whose dimension is $(n-1)(n-2) / 2$, then $K$ leaves invariant one and only 1 dimensional subspace in an $n$-dimensional vector space (cf. Obata [5]).

We can see that for $n(n+1) / 2 \geq r>n(n-1) / 2+1$ the full-isometry group of $M$ contains no subgroup of $r$ whose isotropy subgroup is compact. In fact, suppose that for $n(n+1) / 2 \geq r>n(n-1) / 2+1$ there exists an $r$-dimensional subgroup $G$ whose isotropy subgroup $H_{p}$ is compact for some $p \in M$. Then $\operatorname{dim} H_{p} \leq(n-1)(n-2) / 2$ from Lemma 1. On the other hand, we have

$$
\operatorname{dim} H_{p} \geq \operatorname{dim} G-\operatorname{dim} M>n(n-1) / 2+1-n=(n-1)(n-2) / 2
$$

which is a contradiction to the fact that $\operatorname{dim} H_{p} \leq(n-1)(n-2) / 2$.

We can also derive the following proposition from Lemma 1.

PROPOSITION. If $M$ admits a connected isometry group $G$ of dimension $n(n-1) / 2+1$ whose isotropy subgroup $H_{p}$ is compact for every $p \in M$, then $G$ is transitive on $M$.

Proof. Assume that $G$ is not transitive on $M$. Then the orbit of $G$ through $p$ is of dimension less than $n$. Hence,

$$
\operatorname{dim} H_{p} \geq \operatorname{dim} G-(n-1)=(n-1)(n-2) / 2+1
$$

which is impossible from Lemma 1.

Hereafter, let $G$ be a connected isometry group of dimension $n(n-1) / 2+1$ whose isotropy subgroup $H_{p}$ is compact for every $p \in M$. From the proposition, $\operatorname{dim} H_{p}=(n-1)(n-2) / 2$ and we may assume that $M=G / H_{p}$.

Let $\mathfrak{g}$ and $\mathfrak{h}$ be the Lie algebras of $G$ and $H:=H_{o}$ respectively. But the use of an $\operatorname{Ad}(H)$-invariant positive definite inner product on $\mathfrak{g}$ which exists from the compactness of $H$, we have a decomposition $\mathfrak{g}=\mathfrak{h}+\mathfrak{m}$ (direct sum) of $\mathfrak{g}$ where $\operatorname{Ad}(H) \mathfrak{m} \nsubseteq \mathfrak{m}$. Let $\pi: G \rightarrow G / H$ be the natural projection. We identify the tangent space $T_{o} M$ at $o:=\pi(H)$ and $\mathfrak{m}$ by $d \pi$. Then the linear isotropy group $\tilde{H}$ acting on $T_{o} M$ corresponds under $d \pi$ to $\operatorname{Ad}(H)$ on $\mathfrak{m}$. The Lorentz inner product $T_{o} M$ induces the Lorentz inner product $\langle,\rangle_{\mathfrak{m}}$ on $\mathfrak{m}$ so that $d \pi: \mathfrak{m} \rightarrow T_{o} M$ is a linear isometry. We note that the inner product $\langle,\rangle_{\mathfrak{m}}$ on $\mathfrak{m}$ is $\operatorname{Ad}(H)$-invariant.

We define the Lorentz inner product $B$ on $\mathfrak{g}$ such that

$$
B(\mathfrak{h}, \mathfrak{m})=0, \quad B \uparrow_{\mathfrak{m}}=\langle,\rangle_{\mathfrak{m}},
$$

and $B \uparrow_{h}$ is positive definite. We extend $B$ to the $G$-left invariant Lorentz metric on $G$ which is denoted by the same letter $B$. Then $(G, B)$ is a Lorentz manifold and $\pi:(G, B) \rightarrow(M,\langle\rangle$,$) is a semi-Riemannian submersion (see O'Neill [6, p. 212]).$

Since $\operatorname{Ad}(H)$ acts on $\mathfrak{m}$ as a compact linear isometry group and $\operatorname{dim} \operatorname{Ad}(H)=$ $(n-1)(n-2) / 2$, it follows from Lemma 1 that there exists one and only one 1dimensional subspace $\mathfrak{m}_{1}$ of $\mathfrak{m}$ which is invariant by $\operatorname{Ad}(H)$. Furthermore, we have 
LEMMA 2. $\mathfrak{m}_{1}$ is timelike.

Putting $\mathfrak{m}_{2}=\left\{X \in \mathfrak{m} ; B\left(X, \mathfrak{m}_{1}\right)=0\right\}$, we have a direct sum decomposition $\mathfrak{m}=\mathfrak{m}_{1}+\mathfrak{m}_{2}$ of $\mathfrak{m}$ where $\left[\mathfrak{h}, \mathfrak{m}_{1}\right]=(0),\left[\mathfrak{h}, \mathfrak{m}_{2}\right]=\mathfrak{m}_{2}$, and the adjoint representation of $\mathfrak{h}$ in $\mathfrak{m}_{2}$ is irreducible. Thus we have a decomposition $\mathfrak{g}=\mathfrak{m}_{1}+\mathfrak{m}_{2}+\mathfrak{h}$ of $\mathfrak{g}$ by $\operatorname{ad}(\mathfrak{h})$, where $\left[\mathfrak{h}, \mathfrak{m}_{1}\right]=(0)$ and $\left[\mathfrak{h}, \mathfrak{m}_{2}\right]=\mathfrak{m}_{2}$ (see Obata $[\mathbf{5}]$ ).

LEMMA 3 (OBATA [5]). We have the following possibilities provided $n \geq 5$ :

(i) $\left[\mathfrak{h}, \mathfrak{m}_{1}\right]=(0),\left[\mathfrak{m}_{1}, \mathfrak{m}_{2}\right]=(0),\left[\mathfrak{m}_{2}, \mathfrak{m}_{2}\right]=(0)$;

(ii) $\left[\mathfrak{h}, \mathfrak{m}_{1}\right]=(0),\left[\mathfrak{m}_{1}, \mathfrak{m}_{2}\right]=(0),\left[\mathfrak{m}_{2}, \mathfrak{m}_{2}\right]=\mathfrak{h}$;

(iii) $\left[\mathfrak{h}, \mathfrak{m}_{1}\right]=(0),\left[\mathfrak{m}_{1}, \mathfrak{m}_{2}\right]=\mathfrak{m}_{2},\left[\mathfrak{m}_{2}, \mathfrak{m}_{2}\right]=(0)$, and $[X, Y]=L(X) Y$ for any $X \in \mathfrak{m}_{1}$ and $Y \in \mathfrak{m}_{2}$ where $L$ is the linear function on $\mathfrak{m}_{1}$ such that $L(X) \neq 0$ for any nonzero $X \in \mathfrak{m}_{1}$.

REMARK 3. For a unit vector $E \in \mathfrak{m}_{1}$ (i.e. $B(E, E)=-1$ ) we put $c:=L(E)$. We may assume $c>0$.

We set $\mathfrak{g}^{\prime}=\mathfrak{h}+\mathfrak{m}_{2}$ and $B^{\prime}=B \uparrow_{\mathfrak{g}^{\prime}}$. Since $B^{\prime}$ is positive definite and $\left.\operatorname{ad}(\mathfrak{h})\right|_{\mathfrak{m}_{2}}=$ $o(n-1)$, there is a basis $\left\{X_{i}, X_{j k}\right\}(1 \leq i \leq n-1,1 \leq j<k \leq n-1)$ of $\mathfrak{g}^{\prime}$ such that

(1) $\left\{X_{i}\right\}$ (resp. $\left.\left\{X_{j k}\right\}\right)$ is a basis of $\mathfrak{m}_{2}$ (resp. $\mathfrak{h}$ ),

(2) $B^{\prime}\left(X_{k}, X_{j}\right)=\delta_{i j}$,

(3) $\left[X_{i j}, X_{k}\right]=\delta_{i k} X_{j}-\delta_{j k} X_{i}(1 \leq i<j \leq n-1,1 \leq k \leq n-1)$.

Then

LEMMA 4 (OBATA [5]). In case (ii) in Lemma 3, there exists a nonzero constant $\alpha$ such that $\left[X_{i}, X_{j}\right]=\alpha X_{i j}(1 \leq i<j \leq n-1)$.

3. From Lemma $3, \mathfrak{m}_{1}$ (resp. $\mathfrak{m}_{2}$ ) induces an integrable $G$-invariant 1- (resp. $n-1-$ ) dimensional distribution $T_{1}$ (resp. $T_{2}$ ) on $M$ such that at each point $p$ of $M, T_{p} M=T_{1}(p)+T_{2}(p),\left\langle T_{1}(p), T_{2}(p)\right\rangle=0$, and $T_{1}(p)$ (resp. $\left.T_{2}(p)\right)$ is timelike (resp. spacelike)

Now, we assume that $M$ is simply connected.

LEMMA 5. When $M$ is simply connected, $\xi:=d \pi(E)$ is well defined on $M$.

ProOF. We will show that for each $p \in M, \xi(p)=d \pi(E(g))=d \tau_{g} d \pi(E(e))$ is independent of the choice of $g \in G$ such that $g o=p$, where $e$ is the identity of $G, o=\pi(H)$, and $\tau_{g}$ is the map: $x \rightarrow g x$ on $M$.

Let $g_{1} o=g_{2} o=p\left(g_{1}, g_{2} \in G\right)$. $G$ being connected, there exist curves $\tilde{g}_{i}:[0,1] \rightarrow$ $G$ such that $\tilde{g}_{i}(0)=e$ and $\tilde{g}_{i}(1)=g_{i}(i=1,2)$. Set $c_{i}(t):=\tilde{g}_{i}(t) o(i=1,2) . M$ being simply connected, $M$ is time orientable. So there exists a unit timelike vector field $X$ on $M$. Then we can see that

$$
\left\langle X\left(c_{i}(t)\right), d \tau_{\tilde{g}_{i}(t)} \xi(o)\right\rangle \neq 0 \quad \text { for any } t \in[0,1] .
$$

The map: $t \rightarrow\left\langle X\left(c_{i}(t)\right), d \tau_{\tilde{g}_{i}(t)} \xi(o)\right\rangle$ being continous, if $\langle X(o), \xi(o)\rangle<0$ (resp. $>0)$, then $\left\langle X(p), d \tau_{g_{i}} \xi(o)\right\rangle<0$ (resp. $>0$ ). Thus $d \tau_{g_{1}} \xi(o)$ and $d \tau_{g_{2}} \xi(o)$ belong to the same connected component of the time cone in $T_{p} M$. Furthermore, $d \tau_{g_{1}} \xi(o)$ and $d \tau_{g_{2}} \xi(o)$ belong to $T_{1}(p)$. Therefore $d \tau_{g_{1}} \xi(o)=d \tau_{g_{2}} \xi(o)$.

We define the nonzero 1 -form $\omega$ on $M$ by $\omega(X):=\langle X, \xi\rangle$. Then we can see $\omega$ is closed. Since $M$ is simply connected there exists a $C^{\infty}$ function $f: M \rightarrow \mathbf{R}$ such that $d f=\omega$. For each $a \in f(M), f^{-1}(a)$ is a closed spacelike hypersurface 
of $M$ and each connected component of $f^{-1}(a)$ is a leaf of $T_{2}$. Since $\nabla_{\xi} \xi=0$ and $\xi=d \pi(E)$, each integral curve of $\xi$ which is a leaf of $T_{1}$ is a complete geodesic. For a point $p \in M$, let $\gamma_{p}(t)$ be the integral curve of $\xi$ such that $\gamma_{p}(0)=p$. Then we have easily that $f\left(\gamma_{p}(t)\right)=-t+a$ for $p \in f^{-1}(a)$. Therefore $f(M)=\mathbf{R}$. Let $N$ be $f^{-1}(0)$ and $N_{0}$ be a connected component of $N$. We define the map $F: \mathbf{R} \times N_{0} \rightarrow M$ by $F(t, x):=\gamma_{x}(t)=\operatorname{Exp}(t \xi(x))$. Then we have

LEMMA 6. F is the onto diffeomorphism.

Proof. Assume that $F(t, x)=F\left(t^{\prime}, x^{\prime}\right)$. We have

$$
t=-f\left(\gamma_{x}(t)\right)=-f(F(t, x))=-f\left(F\left(t^{\prime}, x^{\prime}\right)\right)=t^{\prime} .
$$

Since geodesics $\gamma_{x}$ and $\gamma_{x^{\prime}}$ are leaves of $T_{1}$ through $F(t, x)=F\left(t^{\prime}, x^{\prime}\right)$ and $t=t^{\prime}$, we have $x=x^{\prime}$. Thus $F$ is one-to-one. It is evident that $F$ is differentiable. Set $M_{0}:=F\left(\mathbf{R} \times N_{0}\right)$. Then $M_{0}$ is open in $M$. It remains to be shown that $M_{0}$ is closed in $M$. Suppose that $F\left(t_{k}, x_{k}\right)=p_{k}$ is a sequence approaching some point $q$ in $M$. Let $\tilde{f}: \mathbf{R} \rightarrow \mathbf{R}$ be the function defined by $\tilde{f}(t):=f(F(t, x))$ for some $x \in N_{0}$. Then $\tilde{f}$ is independent of the choice $x \in N_{0}$, for $\tilde{f}(t)=-t$. Since $\tilde{f}^{-1}\left(f\left(p_{k}\right)\right)=t_{k}$ and $\tilde{f}^{-1}\left(f\left(p_{k}\right)\right) \rightarrow \tilde{f}^{-1}(f(q))$, we have $t_{k} \rightarrow t_{0}:=\tilde{f}^{-1}(f(q))$. Letting $x:=\gamma_{q}\left(-t_{0}\right)=\operatorname{Exp}\left(-t_{0} \xi(q)\right)$, we have

$$
x_{k}=\gamma_{p_{k}}\left(-t_{k}\right)=\operatorname{Exp}\left(-t_{k} \xi\left(p_{k}\right)\right) \rightarrow \gamma_{q}\left(-t_{0}\right) .
$$

Since $N_{0}$ is closed, $x$ belongs to $N_{0}$ so that $q=F\left(t_{0}, x\right) \in M_{0}$. Thus $M=M_{0}$; furthermore, $N=N_{0}$.

Since $M$ is homogeneous and $T_{2}$ is $G$-invariant, $N$ is a homogeneous Riemannian manifold so that $N$ is complete. Furthermore, $N$ is simply connected, because $M$ which is diffeomorphic to $\mathbf{R} \times N$, is simply connected. Therefore, in cases (i) and (iii) in Lemma $3, N$ is isometric to the Euclidean space $\mathbf{E}^{n-1}$. In case (ii) in Lemma $3, N$ is isometric to a sphere $S^{n-1}$ or a hyperbolic space $\mathbf{H}^{n-1}$ by Lemma 4.

Let $\left(U, \phi=\left(t_{1}, \ldots, t_{n-1}\right)\right)$ be a local coordinate around a point $p$ in $N$. Then $\left(\mathbf{R} \times U, \mathrm{id} \times \phi=\left(t, t_{1}, \ldots, t_{n-1}\right)\right)$ is a local coordinate around $(a, p)$ in $\mathbf{R} \times N$. Let $\tilde{U}:=F(\mathbf{R} \times U)$ and define $\tilde{\phi}: \tilde{U} \rightarrow \mathbf{R}^{n}$ by $(\mathrm{id} \times \phi) \circ F^{-1}$. Then $(\tilde{U}, \tilde{\phi}=$ $\left.\left(x_{0}, x_{1}, \ldots, x_{n-1}\right)\right)$ is a local coordinate around $\tilde{p}=F(a, p)$ in $M$. We can see that $d F(\partial / \partial t)=\xi=\partial / \partial x_{0} \in T_{1}$ and $d F\left(\partial / \partial t_{i}\right)=\partial / \partial x_{i} \in T_{2}(i=1, \ldots, n-1)$. So we have $\left\langle\partial / \partial x_{0}, \partial / \partial x_{0}\right\rangle=-1$ and $\left\langle\partial / \partial x_{0}, \partial / \partial x_{i}\right\rangle=0$ for $1 \leq i \leq n-1$.

LEMMA 7. In cases (i) and (ii) in Lemma 3, F: $\left(\mathbf{R} \times N,-d t^{2}+d s_{N}^{2}\right) \rightarrow$ $(M,\langle\rangle$,$) is isometry, where d s_{N}^{2}$ is the metric of $N$.

Proof. It is enough to show that for $1 \leq i, j \leq n-1,\left\langle\partial / \partial x_{i}, \partial / \partial x_{j}\right\rangle$ is independent of $x_{0}$. Since $\pi:(G, B) \rightarrow(M,\langle\rangle$,$) is the semi-Riemannian submersion, it$ follows from Lemma 3 that $T_{1}$ is parallel. So, for $1 \leq i, j \leq n-1$, we have

$$
\begin{aligned}
& \left(\partial / \partial x_{0}\right)\left\langle\partial / \partial x_{i}, \partial / \partial x_{j}\right\rangle=\left\langle\nabla_{\partial / \partial x_{i}} \partial / \partial x_{0}, \partial / \partial x_{j}\right\rangle \\
& +\left\langle\partial / \partial x_{i}, \nabla \partial / \partial x_{j} \partial / \partial x_{0}\right\rangle \\
& =0
\end{aligned}
$$

because $\nabla_{\partial / \partial x_{i}} \partial / \partial x_{0}$ and $\nabla_{\partial / \partial x_{j}} \partial / \partial x_{0}$ belong to $T_{1}$. 
LEMMA 8. In case (iii) in Lemma $3, F:\left(\mathbf{R} \times N,-d t^{2}+\exp (-2 c t) d s^{2}\right) \rightarrow$ $(M,\langle\rangle$,$) is isometry, where d s^{2}$ is the flat metric of $N=\mathbf{E}^{n-1}$.

ProOF. Since $\pi:(G, B) \rightarrow(M,\langle\rangle$,$) is the semi-Riemannian submersion, the$ equalities $\nabla_{\partial / \partial x_{j}} \partial / \partial x_{0}=-c\left(\partial / \partial x_{j}\right)(1 \leq j \leq n-1)$ follows from Lemma 3 . We have

$$
\left(\partial / \partial x_{0}\right)\left\langle\partial / \partial x_{i}, \partial / \partial x_{j}\right\rangle=-2 c\left\langle\partial / \partial x_{i}, \partial / \partial x_{j}\right\rangle
$$

for $1 \leq i, j \leq n-1$ so that we have

$$
\left\langle\partial / \partial x_{i}, \partial / \partial x_{j}\right\rangle=\exp \left(-2 c x_{0}\right) g_{i j}\left(x_{1}, \ldots, x_{n-1}\right) .
$$

Thus

$$
F^{*}\langle,\rangle=-d t^{2}+\exp (-2 c t) \sum_{i, j=1}^{n-1} g_{i j} \circ F\left(t_{1}, \ldots, t_{n-1}\right) d t_{i} d t_{j} .
$$

Since $N$ is isometric to $\mathbf{E}^{n-1}$, we may assume that $g_{i j}=\delta_{i j}$.

PROOF OF THEOREM. Consider case (i) in Lemma 3. If $M$ is simply connected, $M$ is isometric to $\left(\mathbf{R} \times \mathbf{E}^{n-1},-d t^{2}+d s^{2}\right)$ by Lemma 7 where $d s^{2}$ is the metric of $\mathbf{E}^{n-1}$. To find a non-simply-connected $M$, we must look for a discrete subgroup $\Gamma$ of the full isometry group of $\mathbf{R} \times \mathbf{E}^{n-1}$ which commutes with $G=\mathbf{R} \times I^{0}\left(\mathbf{E}^{n-1}\right)$ elementwise. It is easy to verify that $\Gamma$ is generated by a translation of $\mathbf{R}$. Hence, if $M$ is not simply connected, then $M$ is isometric to $S^{1}(r) \times \mathbf{E}^{n-1}$.

Consider case (ii) in Lemma 3. If $M$ is simply connected, $M$ is isometric to $\left(\mathbf{R} \times N,-d t^{2}+d s_{N}^{2}\right)$ by Lemma 7 where $N$ is $S^{n-1}\left(r^{\prime}\right)$ or $\mathbf{H}^{n-1}\left(r^{\prime}\right)$. By the same method as above, if $M$ is not simply connected, then $M$ is isometric to $S^{1}(r) \times S^{n-1}\left(r^{\prime}\right), \mathbf{R} \times \mathbf{P}^{n-1}, S^{1}(r) \times \mathbf{P}^{n-1}$ or $S^{1}(r) \times \mathbf{H}^{n-1}\left(r^{\prime}\right)$.

Consider case (iii) in Lemma 3 . If $M$ is simply connected, $M$ is isometric to $\left(\mathbf{R} \times \mathbf{R}^{n-1},-d t^{2}+\exp (-2 c t) \sum_{j=1}^{n-1} d t_{j}^{2}\right)$ by Lemma 8 . This space is isometric to the space $(\mathrm{v})$ in the Example by the transformation

$$
\mathbf{R} \times \mathbf{R}^{n-1} \ni\left(t, t_{1}, \ldots, t_{n-1}\right) \rightarrow\left(t_{1}, \ldots, t_{n-1}, e^{c t} / c\right) \in U_{n}^{+} .
$$

A discrete subgroup of the full isometry group $I\left(U_{n}^{+}\right)$which commutes with $G=$ $I^{0}\left(U_{n}^{+}\right)$elementwise consists of the identity element.

The proof of the theorem is complete.

ACKNOWLEDGMENT. The author would like to thank Professor Y. Maeda for suggesting the problem. The author also would like to thank Professors H. Kitahara, S. Yorozu, and M. Obata for their helpful advice and encouragement, and the referee for his kind and suitable advice.

\section{REFERENCES}

1. S. Ishihara, Homogeneous Riemannian spaces of four dimensions, J. Math. Soc. Japan 7 (1955), 345-370.

2. S. Kobayashi, Transformation groups in differential geometry, Springer-Verlag, Berlin, 1972.

3. H. Matsuda, A note on an isometric imbedding of upper half-space into anti-de-Sitter space, Hokkaido Math. J. 13 (1984), 123-132.

4. K. Nomizu, The Lonentz-Poincaré metric on upper half-space and its extension, Hokkaido Math. J. 11 (1982), 253-261.

5. M. Obata, On n-dimensional homogeneous spaces of Lie groups of dimension greater than $n(n-1) / 2$, J. Math. Soc. Japan 7 (1955), 371-388. 
6. B. O'Neill, Semi-Riemannian geometry with applications to relativity, Academic Press, New York, 1983.

7. J. Wolf, Spaces of constant curvature, Publish or Perish, Boston, Mass., 1984.

8. K. Yano, On n-dimensional Riemannian spaces admitting a group of motions of onder $n(n-1) / 2+1$, Trans. Amer. Math. Soc. 74 (1953), 260-279.

Department of Mathematics, KanazaWA Medical University, UChinadaMACHI, ISHIKAWA-KEN, 920-02, JAPAN 